\title{
Revisiting Liebmann's theorem in higher codimension
}

\author{
by \\ Jogli G. ARAÚJO and Henrique F. DE LIMA \\ Presented by Tadeusz JANUSZKIEWICZ
}

Summary. We deal with compact surfaces immersed with flat normal bundle and parallel normalized mean curvature vector field in a space form $\mathbb{Q}_{c}^{2+p}$ of constant sectional curvature $c \in\{-1,0,1\}$. Such a surface is called an $L W$-surface when it satisfies a linear Weingarten condition of the type $K=a H+b$ for some real constants $a$ and $b$, where $H$ and $K$ denote the mean and Gaussian curvatures, respectively. In this setting, we extend the classical rigidity theorem of Liebmann (1899) showing that a non-flat LW-surface with non-negative Gaussian curvature must be isometric to a totally umbilical round sphere.

1. Introduction and statement of the main result. The study of surfaces immersed in a 3-dimensional Riemannian space form $\mathbb{Q}_{c}^{3}$ of constant sectional curvature $c$ plays an important role in the theory of submanifolds. In relation to this topic, in 1897 Hadamard [3] proved that an ovaloid, that is, a compact connected surface with positive Gaussian curvature, in the 3 -dimensional Euclidean space $\mathbb{R}^{3}$ is a topological sphere. In view of this result, it was natural to look for conditions which allowed one to conclude that such a surface was necessarily a totally umbilical round sphere. In 1899 Liebmann [5] obtained his celebrated rigidity result, which states that every compact connected surface in $\mathbb{R}^{3}$ with constant Gaussian curvature is necessarily a totally umbilical round sphere.

Later on, there have been different generalizations of Liebmann's theorem from several points of view for surfaces, and more generally hypersurfaces, in the Euclidean space [4, 7, 8, 9, 10, or in the hyperbolic space or an open hemisphere [6]. In [1], as an application of the Gauss-Bonnet theorem

2010 Mathematics Subject Classification: Primary 53C42; Secondary 53A10, 53C20.

Key words and phrases: space forms, compact LW-surfaces, parallel normalized mean curvature vector field, totally umbilical round spheres.

Received 29 May 2019.

Published online 17 June 2019. 
along with a formula involving the Gaussian curvatures of the first and second fundamental forms of the surface, Aledo, Alías and Romero established a new direct proof of these results.

Here, we consider a wide class of surfaces $M^{2}$ immersed in a $(2+p)$ dimensional space form $\mathbb{Q}_{c}^{2+p}$ of constant sectional curvature $c \in\{-1,0,1\}$, which extend those of constant Gaussian curvature, the so-called linear Weingarten surfaces or simply $L W$-surfaces. We recall that a surface is said to be an LW-surface when its mean curvature $H$ and its Gaussian curvature $K$ satisfy a linear relation of the type $K=a H+b$ for some constants $a, b \in \mathbb{R}$. These surfaces were originally introduced by Weingarten [11, 12] in the context of the problem of finding all surfaces of the Euclidean space isometric to a prescribed surface of revolution. In this setting, we obtain the following rigidity result which can be regarded as an extension of the previously mentioned ones:

THEOREM 1.1. Let $M^{2}$ be a compact non-flat $L W$-surface immersed in a Riemannian space form $\mathbb{Q}_{c}^{2+p}$ of constant sectional curvature $c \in\{-1,0,1\}$, with flat normal bundle, parallel normalized mean vector field and such that its Gaussian curvature $K$ and mean curvature $H$ satisfy $K=a H+b$ with $a^{2}+2(2 b-c) \geq 0$. If $K$ is non-negative on $M^{2}$, then $M^{2}$ is a totally umbilical round sphere.

The proof of Theorem 1.1 is given in Section 3. Before, in Section 2 we recall some basic facts concerning the geometry of surfaces immersed in a space form.

2. Preliminaries. Let $M^{2}$ be a connected surface immersed in a space form $\mathbb{Q}_{c}^{2+p}$ of constant sectional curvature $c$. We will use the following convention on the range of indices:

$$
1 \leq A, B, C, \ldots \leq 2+p, \quad 1 \leq i, j, k, \ldots \leq 2, \quad 3 \leq \alpha, \beta, \gamma, \ldots \leq 2+p .
$$

We choose a local orthonormal frame field $\left\{e_{1}, e_{2}, e_{3}, \ldots, e_{2+p}\right\}$ along $M^{2}$, where $\left\{e_{i}\right\}_{i=1,2}$ are tangent to $M^{2}$ and $\left\{e_{\alpha}\right\}_{\alpha=3, \ldots, 2+p}$ are normal to $M^{2}$. Let $\left\{\omega_{B}\right\}$ be the corresponding dual coframe, and $\left\{\omega_{B C}\right\}$ the connection 1-forms on $\mathbb{Q}_{c}^{2+p}$. The second fundamental form $h$, the curvature tensor $R$ and the normal curvature tensor $R^{\perp}$ of $M^{2}$ can be given by

$$
\begin{aligned}
\omega_{i \alpha} & =\sum_{j} h_{i j}^{\alpha} \omega_{j}, \quad h=\sum_{i, j, \alpha} h_{i j}^{\alpha} \omega_{i} \otimes \omega_{j} \otimes e_{\alpha}, \\
d \omega_{i j} & =\sum_{k} \omega_{i k} \wedge \omega_{k j}-\frac{1}{2} \sum_{k, l} R_{i j k l} \omega_{k} \wedge \omega_{l}, \\
d \omega_{\alpha \beta} & =\sum_{\gamma} \omega_{\alpha \gamma} \wedge \omega_{\gamma \alpha}-\frac{1}{2} \sum_{k, l} R_{\alpha \beta k l}^{\perp} \omega_{k} \wedge \omega_{l} .
\end{aligned}
$$


Moreover, the components $h_{i j k}^{\alpha}$ of the covariant derivative $\nabla h$ satisfy

$$
\sum_{k} h_{i j k}^{\alpha} \omega_{k}=d h_{i j}^{\alpha}+\sum_{k} h_{k i}^{\alpha} \omega_{k j}+\sum_{k} h_{k j}^{\alpha} \omega_{k i}+\sum_{\beta} h_{i j}^{\beta} \omega_{\beta \alpha} .
$$

The Gauss equation is

$$
R_{i j k l}=c\left(\delta_{i k} \delta_{j l}-\delta_{i l} \delta_{j k}\right)+\sum_{\alpha}\left(h_{i k}^{\alpha} h_{j l}^{\alpha}-h_{i l}^{\alpha} h_{j k}^{\alpha}\right) .
$$

In particular, the components of the Ricci tensor $R_{i k}$ are given by

$$
R_{i k}=c \delta_{i k}+2 \sum_{\alpha} H^{\alpha} h_{i k}^{\alpha}-\sum_{\alpha, j} h_{i j}^{\alpha} h_{j k}^{\alpha},
$$

where $H^{\alpha}=\frac{1}{2} \sum_{i} h_{i i}^{\alpha}$ are the components of the mean curvature vector field $\mathbf{H}=\sum_{\alpha} H^{\alpha} e_{\alpha}$.

From (2.3) we get the relation

$$
2 K=-2+4 H^{2}-S,
$$

where $K$ stands for the Gaussian curvature of $M^{2}, H=|\mathbf{H}|$ is the mean curvature function and $S=|h|^{2}=\sum_{i, j, \alpha}\left(h_{i j}^{\alpha}\right)^{2}$ is the squared norm of the second fundamental form $h$ of $M^{2}$.

Assuming that $M^{2}$ has flat normal bundle (that is, $R^{\perp}=0$ ), by exterior differentiation of (2.1) we obtain the Ricci identity

$$
h_{i j k l}^{\alpha}-h_{i j l k}^{\alpha}=\sum_{m} h_{m j}^{\alpha} R_{m i k l}+\sum_{m} h_{i m}^{\alpha} R_{m j k l} .
$$

Moreover, the Codazzi equation is given by

$$
h_{i j k}^{\alpha}=h_{i k j}^{\alpha}=h_{j i k}^{\alpha} .
$$

3. Proof of Theorem 1.1. In what follows, we will deal with surfaces $M^{2}$ of $\mathbb{Q}_{c}^{2+p}$ having parallel normalized mean curvature vector field, which means that the mean curvature function $H$ is positive and the corresponding normalized mean curvature vector field $\mathbf{H} / H$ is parallel as a section of the normal bundle.

In this context, we can choose a local orthonormal frame $\left\{e_{1}, \ldots, e_{2+p}\right\}$ such that $e_{3}=\mathbf{H} / H$. Thus,

$$
H^{3}=\frac{1}{2} \operatorname{tr}\left(h^{3}\right)=H \quad \text { and } \quad H^{\alpha}=\frac{1}{2} \operatorname{tr}\left(h^{\alpha}\right)=0, \alpha \geq 4,
$$

where $h^{\alpha}$ stands for the $2 \times 2$ matrix $\left(h_{i j}^{\alpha}\right)$.

We will consider the symmetric tensor

$$
\Phi=\sum_{\alpha, i, j} \Phi_{i j}^{\alpha} \omega_{i} \otimes \omega_{j} \otimes e_{\alpha},
$$

where $\Phi_{i j}^{\alpha}=h_{i j}^{\alpha}-H^{\alpha} \delta_{i j}$. Consequently,

$$
\Phi_{i j}^{3}=h_{i j}^{3}-H \delta_{i j} \quad \text { and } \quad \Phi_{i j}^{\alpha}=h_{i j}^{\alpha}, 4 \leq \alpha \leq 2+p .
$$


Let $|\Phi|^{2}=\sum_{i, j, \alpha}\left(\Phi_{i j}^{\alpha}\right)^{2}$ be the square of the length of $\Phi$. From 2.4 , it is not difficult to verify that $\Phi$ is traceless with

$$
|\Phi|^{2}=S-2 H^{2}=2\left(c+H^{2}-K\right) .
$$

In order to prove Theorem 1.1, we will also need the following key lemma which is obtained by just adapting the proof of [13, Proposition 2.2]:

Lemma 3.1. Let $M^{2}$ be an $L W$-surface immersed in $\mathbb{Q}_{c}^{2+p}$, with $K=$ $a H+b$ for some $a, b \in \mathbb{R}$ such that $a^{2}+8(b-c) \geq 0$. Then

$$
|\nabla h|^{2} \geq 4|\nabla H|^{2} \text {. }
$$

Moreover, if equality holds in (3.4) on $M^{2}$, then $H$ is constant on $M^{2}$.

Now, we are in a position to present the proof of Theorem 1.1 .

Proof of Theorem 1.1. We have

$$
\frac{1}{2} \Delta S=\sum_{i, j, \alpha} h_{i j}^{\alpha} \Delta h_{i j}^{\alpha}+\sum_{i, j, k, \alpha}\left(h_{i j k}^{\alpha}\right)^{2},
$$

where the Laplacian $\Delta h_{i j}^{\alpha}$ of $h_{i j}^{\alpha}$ is defined by $\Delta h_{i j}^{\alpha}=\sum_{k} h_{i j k k}^{\alpha}$. Using the Codazzi equation (2.6) in (3.5) we obtain

$$
\frac{1}{2} \Delta S=|\nabla h|^{2}+\sum_{i, j, k, \alpha} h_{i j}^{\alpha} h_{k i j k}^{\alpha} .
$$

Thus, from (2.5), 3.1) and (3.6), we conclude that

$$
\begin{aligned}
\frac{1}{2} \Delta S= & |\nabla h|^{2}+\sum_{i, j} n H_{i j}^{n+1} h_{i j}^{n+1}+\sum_{i, j, m, k, \alpha} h_{i j}^{\alpha} h_{m i}^{\alpha} R_{m k j k} \\
& +\sum_{i, j, k, m, \alpha} h_{i j}^{\alpha} h_{k m}^{\alpha} R_{m i j k} .
\end{aligned}
$$

Consequently, taking a (local) orthonormal frame $\left\{e_{1}, e_{2}\right\}$ on $M^{2}$ such that $h_{i j}^{\alpha}=\lambda_{i}^{\alpha} \delta_{i j}$ for every $\alpha$, from 3.7 we obtain the Simons-type formula

$$
\frac{1}{2} \Delta S=|\nabla h|^{2}+\sum_{i} \lambda_{i}^{3}(2 H)_{i i}+\frac{1}{2} \sum_{i, j, \alpha} R_{i j i j}\left(\lambda_{i}^{\alpha}-\lambda_{j}^{\alpha}\right)^{2} .
$$

We define an appropriate modified Cheng-Yau operator by

$$
L=\square-\frac{1}{2} a \Delta,
$$

where the square operator is defined by

$$
\square f=\sum_{i, j}\left(2 H \delta_{i j}-h_{i j}^{3}\right) f_{i j}
$$

for each $f \in C^{\infty}(M)$. 
Setting $f=2 H$ in 3.10 , we obtain

$$
\begin{aligned}
\square(2 H) & =2 H \Delta(2 H)-\sum_{i} \lambda_{i}^{3}(2 H)_{i i} \\
& =\frac{1}{2} \Delta(2 H)^{2}-\sum_{i}(2 H)_{i}^{2}-\sum_{i} \lambda_{i}^{3}(2 H)_{i i} \\
& =\Delta R+\frac{1}{2} \Delta S-4|\nabla H|^{2}-\sum_{i} \lambda_{i}^{3}(2 H)_{i i} .
\end{aligned}
$$

Consequently, inserting (3.8) into 3.11) we get

$$
\square(2 H)=\Delta R+|\nabla h|^{2}-4|\nabla H|^{2}+\frac{1}{2} \sum_{i, j, \alpha} R_{i j i j}\left(\lambda_{i}^{\alpha}-\lambda_{j}^{\alpha}\right)^{2} .
$$

Since $R=a H+b$, from 3.9 and 3.12 we have

$$
L(2 H)=|\nabla h|^{2}-4|\nabla H|^{2}+\frac{1}{2} \sum_{i, j} R_{i j i j}\left(\lambda_{i}^{\alpha}-\lambda_{j}^{\alpha}\right)^{2} .
$$

From the Gauss equation we have

$$
R_{i j i j}=c+\sum_{\beta} \lambda_{i}^{\beta} \lambda_{j}^{\beta} .
$$

Hence, using (3.14), 2.4 and (3.3) we have

$$
\begin{aligned}
\frac{1}{2} \sum_{i, j, \alpha} R_{i j i j}\left(\lambda_{i}^{\alpha}-\lambda_{j}^{\alpha}\right)^{2} & =\sum_{\alpha} R_{1212}\left(\lambda_{1}^{\alpha}-\lambda_{2}^{\alpha}\right)^{2} \\
& =\left(c+\sum_{\beta} \lambda_{1}^{\beta} \lambda_{2}^{\beta}\right) \sum_{\alpha}\left(\lambda_{1}^{\alpha}-\lambda_{2}^{\alpha}\right)^{2} \\
& =2\left(c+\sum_{\beta}\left(\frac{\left|h^{\beta}\right|^{2}}{2}-\left|\Phi^{\beta}\right|^{2}\right)\right)|\Phi|^{2} \\
& =2\left(c+\frac{S}{2}-|\Phi|^{2}\right)|\Phi|^{2} \\
& =|\Phi|^{2}\left(-|\Phi|^{2}+2 H^{2}+2 c\right)=2 K|\Phi|^{2} .
\end{aligned}
$$

Thus, using Lemma 3.1 and since we are supposing that $K$ is non-negative on $M^{2}$, from 3.13 and 3.15 we get

$$
L(H) \geq K|\Phi|^{2} \geq 0 .
$$

On the other hand, from $(3.9)$ and $(3.10)$ it is not difficult to verify that

$$
L(H)=\operatorname{div}_{M}(P(\nabla H)),
$$

where $P=(2 H+a / 2) I-h^{3}$ and $I$ denotes the identity in the algebra of smooth vector fields on $M^{2}$. 
From (3.16) and (3.17), integrating $L(H)$ on $M^{2}$, which is supposed be compact, we obtain $L(H)=0$ on $M^{2}$. So, returning to (3.13) we get $|\nabla h|^{2}=4|\nabla H|^{2}$ on $M^{2}$. Thus, using once more Lemma 3.1 we conclude that $H$ is constant on $M^{2}$. Consequently, since $K=a H+b$ and $M^{2}$ is also assumed be non-flat, from (3.16) we infer that $|\Phi|$ vanishes identically, and therefore $M^{2}$ is totally umbilical.

Consequently, since $M^{2}$ is totally umbilical and taking into account (3.1), we get

$$
h^{\alpha}=\left\langle\mathbf{H}, e_{\alpha}\right\rangle I=H^{\alpha} I=0
$$

for every $\alpha>3$. This implies that the first normal subspace

$$
N_{1}=\left\{e_{\alpha} \in \mathfrak{X}^{\perp}(M) ; h^{\alpha}=0\right\}^{\perp}
$$

is parallel and has dimension 1. Therefore, we can apply [2, Proposition 4.1] to reduce the codimension of $M^{2}$ to 1 and we conclude that it must be isometric to a totally umbilical round sphere.

Acknowledgements. The first author was partially supported by INCTMat/CAPES, Brazil. The second author was partially supported by CNPq, Brazil, grant 303977/2015-9.

\section{References}

[1] J. A. Aledo, L. J. Alías and A. Romero, A new proof of Liebmann classical rigidity theorem for surfaces in space forms, Rocky Mountain J. Math. 35, (2005), 1811-1824.

[2] M. Dajczer, Submanifolds and Isometric Immersions, Math. Lect. Ser. 13, Publish or Perish, Houston, TX, 1990.

[3] J. Hadamard, Sur certaines propriétés des trajectoires en dynamique, J. Math. Pures Appl. 3 (1897), 331-387.

[4] D. Koutroufiotis, Two characteristic properties of the sphere, Proc. Amer. Math. Soc. 44 (1974), 176-178.

[5] H. Liebmann, Eine neue Eigenschaft der Kugel, Nachr. Königl. Ges. Wiss. Göttingen Math.-Phys. Kl. 1899, 44-55.

[6] S. Montiel and A. Ros, Compact hypersurfaces: The Alexandrov theorem for higher order mean curvatures, in: Differential Geometry, Pitman Monogr. Surveys Pure Appl. Math. 52, Longman Sci. Tech., Harlow, 1991, 279-296.

[7] A. Ros, Compact hypersurfaces with constant higher order mean curvatures, Rev. Mat. Iberoamer. 3 (1987), 447-453.

[8] A. Ros, Compact hypersurfaces with constant scalar curvature and a congruence theorem, J. Differential Geom. 27 (1988), 215-220.

[9] R. Schneider, Closed convex hypersurfaces with second fundamental form of constant curvature, Proc. Amer. Math. Soc. 35 (1972), 230-233.

[10] U. Simon, Characterizations of the sphere by the curvature of the second fundamental form, Proc. Amer. Math. Soc. 55 (1976), 382-384.

[11] J. Weingarten, Ueber eine Klasse auf einander abwickelbarer Flächen, J. Reine Angew. Math. 59 (1861), 382-393. 
[12] J. Weingarten, Ueber die Flächen, derer Normalen eine gegebene Fläche berühren, J. Reine Angew. Math. 62 (1863), 61-63.

[13] D. Yang and Z. Hou, Linear Weingarten spacelike submanifolds in de Sitter space, J. Geom. 103 (2012), 177-190.

Jogli G. Araújo

Departamento de Matemática

Universidade Federal Rural de Pernambuco

52.171-900 Recife, Pernambuco, Brazil

E-mail: jogli.silva@ufrpe.br
Henrique F. de Lima (corresponding author) Departamento de Matemática Universidade Federal de Campina Grande 58.429-970 Campina Grande, Paraíba, Brazil E-mail: henrique@mat.ufcg.edu.br 
